\title{
DNS and LES of Turbulent Channel Flow with Hydrophobic Surface
}

\author{
X. L. Yang ${ }^{1 *}$, G. W. He ${ }^{2}$, X. Zhang ${ }^{3}$ \\ LNM, Institute of Mechanics, Chinese Academy OF Sciences, Beijing 100080, China \\ Email: ${ }^{1}$ yxiaolei@lnm.imech.ac.cn, ${ }^{2}$ hgw@lnm.imech.ac.cn, ${ }^{3}$ zhangx@lnm.imech.ac.cn
}

\begin{abstract}
Hydrophobic surface benefits for drag reduction. Min and Kim[1] do the first Direct Numerical Simulation on drag reduction in turbulent channel flow. And Fukagata and Kasagi[2] make some theoretical analysis based on Dean 3 's formula and some observations in the DNS results. Using their theory, they conclude that drag reduction is possible in large Reynolds number. Both Direct Numerical Simulation (DNS) and Large Eddy Simulation (LES) are performed in our research. How the LES behaving in the turbulent channel flow with hydrophobic surface is examined. Original Smagorinsky model and its Dynamical model are used in LES. The slip velocities predicted by LES using Dynamical model are in good agreement with DNS as shown in the Figure. Although the percentage of drag reduction predicted by LES shows some discrepancies, it is in the error limit for industrial flow. First order and second order moments of LES are also examined and compared with DNS's results. The first-order moments is calculated well by LES. But there are some discrepancies of second-order moments between LES and DNS.
\end{abstract}

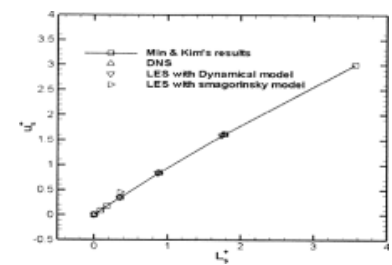

Figure: The slip velocity normalized by the actual shear velocity, $L_{s}^{+}$is normalized by the no slip shear velocity respectively

\section{REFERENCES}

1. Min T., Kim J. Effects of hydrophobic surface on skin-friction drag. Phys. Fluids, 2004;16: L55

2. Fukagata K., Kasagi N. A theoretical prediction of friction drag reduction in turbulent flow by superhydrophobic surfaces. Phys. Fluids, 2006;18: 051703-1

3. Dean R. B. Reynolds number dependency of skin friction and other bulk flow variables in twodimensional rectangular duct flow. ASME Trans. J. Fluids Eng., 1978;100:215 